\title{
VIVES, VITORIA Y MARIANA: RELIGIÓNY PROGRESO SOCIAL
}

\section{VIVES VITORIA AND MARIANA: THREE EXAMPLES OF CA- THOLIC SOCIAL THOUGHT}

\author{
Mira de Orduña, J.M., Vilarroig, J. and Monfort, J.M. \\ Universidad de CEU Cadernal Herrera (España)
}

Resumen: Se proponen tres ejemplos históricos para mostrar cómo la religión, en la forma del cristianismo católico, ha promovido el avance de la filosofía moral o la filosofía social. Frente a posiciones que entienden que la religión tiene como función principal la conservación del status quo de una sociedad en particular (Marx o Durkheim) estos casos notables muestran que más bien es al contrario: las motivaciones religiosas han provocado cambios en la forma de pensar que inevitablemente implican cambios en el modo de vida (Weber). Elegimos tres autores ligados por la época y el espacio (Siglo de oro español) y la religión (cristianismo católico). Juan Luis Vives propuso desde la filosofía cristiana que inspira todo su trabajo una asistencia regular y organizada a los pobres, que andando el tiempo se convertiría en la asistencia social contemporánea. Francisco de Vitoria, reflexionando sobre las causas de la guerra justa, los títulos de conquista o esclavitud, sentó las bases del derecho internacional al margen de la imposición violenta. Juan de Mariana hizo una reflexión política limitando tanto el poder del emperador que incluso sugirió la posibilidad del tiranicidio en caso de necesidad. Estos tres autores nos muestran que, aunque la religión continúa jugando un papel importante en la transmisión del estado de cosas, también ejerce una influencia que permite la evolución en las costumbres y, en consecuencia, en la reflexión ética.

\section{Palabras clave: VIVES; VITORIA; MARIANA; ÉTICA; RELIGIÓN}

Abstract: It is intended to show with three historical examples how religion, in the form of Catholic Christianity, has been decisive in advancing moral philosophy or social philosophy. Faced with positions that understand that religion has as its main function the conservation of the status quo of a particular society (Marx or Durkheim), we want to show with some notable cases that it is rather the opposite: religious motivations have brought about changes in the way of thinking that inevitably entail changes in the way of life (Weber). We choose three authors encompassed within the same period and the same geographical area (the Spanish Renaissance). Francisco de Vitoria, reflecting on the causes of just war, the titles of conquest or slavery, laid the foundations of international law outside violent imposition. Juan Luis Vives proposed, from the Christian philosophy that inspires all his work, a regular and organized assistance to the poor, which would be the basis of contemporary social assistance. Juan 
de Mariana made a political reflection that limited so much the power of the emperor that he even suggested the possibility of tyrannicide in case of absolute necessity. These three authors show us that, although religion continues to play an important role in transmitting values, it also exerts a beneficial influence that allows evolution in the customs and, consequently, in the philosophical reflection of them.

Key words: VITORIA; VIVES; MARIANA; ETHICS; RELIGION

\section{Introducción}

En este trabajo se van a presentar las tesis más famosas de tres autores conocidos: el abordaje planificado y estatal de la cuestión de la pobreza por parte de Juan Luis Vives la propuesta de un derecho internacional basado en el derecho natural y no en la ley del más fuerte por parte de Vitoria, y la resistencia al tirano hasta el extremo de acabar con su vida por parte de Mariana. Aunque estos tres autores y sus ideas son bien conocidos aquí los vamos a presentar con un doble objetivo: por un lado mostrar que la historia no sólo se mueve por interese materiales sino que también lo hace por intereses espirituales; por otro lado, que la religión no es sólo una fuerza conservadora sino también progresiva y posibilitadora de cambios sociales.

La explicación de fenómenos históricos es siempre compleja. Desde determinada historiografía se pretende que las determinaciones materiales de la existencia eran el auténtico motor de la historia. Bien conocida es la tesis marxiana según la cual la estructura económica de una sociedad determina la superestructura ideológica de la misma (cultura, arte, religión, derecho, moral, etc.) ${ }^{1}$. Y sin embargo no han faltado autores como Weber que contradicen o al menos matizan esta tesis mostrando, por ejemplo, cómo la determinada conciencia religiosa de un pueblo puede conducir a este a profundos cambios económicos ${ }^{2}$.

También desde la filosofía de la historia marxiana se tiende a presentar a la religión como una fuerza regresiva o por lo menos esencialmente conservadora. Desde otras perspectivas, en los últimos años se han multiplicado trabajos divulgativos que presentan la religión como algo pernicioso para la humanidad ${ }^{3}$. Y sin embargo la religión no es sólo una fuerza regresiva sino que también es una fuerza progresiva. Hay abundan-

[1] Marx, C., Prólogo a la Contribución a la crítica de la economía política, en Marxists Internet Archive, marzo de 2001, en https://www.marxists.org/espanol/m-e/1850s/criteconpol.htm

[2] Weber, M., La ética protestante y el espíritu del capitalismo. Madrid: Akal, 1998.

[3] Hitchens, C., Dios no es bueno. Barcelona: Debate, 2009; Dawkins, R., El espejismo de Dios. Madrid: Espasa, 2007; Harris, S., El fin de la fe. Religión, terror y futuro de la razón. Madrid: Paradigma, 2007. 
tes ejemplos que demuestran que propuestas concretas de humanidad han sido propuestas por personas religiosas no a pesar de su religión sino precisamente porque eran religiosas. La atención planificada del pauperismo (Vives), el derecho natural más allá de la fuerza (Vitoria) o la muerte del tirano (Mariana) son elementos ideológicos progresivos que difícilmente podrían atribuirse al pensamiento reaccionario.

Las razones por las que se han escogido estos tres autores y no otros son varias. En primer lugar y más importante para lo que sigue los tres profesan la misma religión católica, que como veremos forma parte del entramado de argumentaciones que les llevan a defender sus respectivas tesis. Los tres comparten el mismo humus cultural por pertenecen al renacimiento o siglo de oro español. Además son tres autores ligados por la misma época: desde el nacimiento del primero (1486) hasta la muerte del último (1623) pasan 137 años. Por último los tres autores comparten un mismo espacio geográfico: aunque nacen en España, se forman entre España y Europa lo cual les da una fortísima impronta internacional.

\section{Juan Luis Vives y el socorro de los pobres}

Juan Luis Vives nació en Valencia en 1492, de familia culta pero sin grandes fortunas. Comenzó la formación académica en su ciudad y pronto pudo ir a la Sorbona parisina para dedicarse a la filosofía, a la teología, al derecho, a la retórica y a las bellas artes. El entusiasmo inicial con el tiempo se convirtió en desencanto y Vives, en nombre del humanismo, se enfrentó al dogmatismo, la pedantería y la falta de criterio de los grandes representantes de la educación del país galo. Su decepción fue máxima y su crítica al sistema educativo de entonces audaz y profunda como demuestra en obras como De tradendis disciplinis o Tratado de la enseñanza.

El clima bélico de principios del siglo XVI provocó su salida hacia Flandes, Brujas se convirtió en su casa y Lovaina en su universidad. Hasta el enfrentamiento del Enrique VIII con el Papa se encargó de la educación de María Tudor en Inglaterra y también de una cátedra en Oxford a lo que tuvo que renunciar con la ruptura de la Iglesia anglicana. En 1540 murió en Brujas, en sus últimos años optó por una vida sencilla e independiente en tierra flamenca y renunció a volver a España para suceder a Nebrija en la universidad de Alcalá ${ }^{4}$.

[4] Para un breve resumen de la vida y obra de Vives puede leerse Guy, A. Los filósofos españoles de ayer y de hoy. Buenos Aires: Losada, 1966, p. 51-57. Para un estudio más amplio y recomendable ver Abellán, J. L., Historia crítica del pensamiento español Vol. II. Madrid: Espasa-Calpe, 1979, pp. 108-133. 
Si bien la obra de Vives es extensa e imponente, no menos llamativa es su persona. Tanto Ortega y Gasset ${ }^{5}$ como Gregorio Marañón, dos de los grandes intelectuales hispanos de siglo XX, han destacado de Vives no tanto sus libros sino cómo entendió y desarrolló su propia vida: «Su obra es, sí, muy vasta y está llena de sabiduría, de esfuerzo generoso y de presentimientos geniales. Pero lo importante de Vives, fue él mismo: su vivir y cómo lo apuró» ${ }^{6}$.

¿Qué podemos destacar de la vida de este valenciano que nos permita entender mejor el sentido de sus trabajos? Vives otorgó a la sabiduría y a la cultura un carácter eminentemente servicial, el objetivo es servir al bien de los pueblos, al bien común. Defendió una idea de humanista eminentemente humilde en lugar de soberbio, rompiendo con los moldes educativos establecidos en París. Tuvo una concepción muy igualitaria de los hombres y un gran sentido de su dignidad. Enunció como máxima: "Homo homini par»" , el hombre es igual al hombre, y así en Escolta del alma se lee: "Que ningún hombre se encarame encima de ningún otro hombre, ni le menosprecie, ni le mire con altanería, ni se prefiera a otro, puesto que todos fuimos enviados a esta vida por Dios, nuestro Padre común, y creados por el mismo derecho» ${ }^{8}$. Como afirma Alfonso Maestre, para Vives el amor es la raíz de toda virtud, «amor a nuestros semejantes que ha de reflejarse en una convivencia regida por la veracidad, el respeto, la solidaridad, la ayuda mutua y la perfecta concordia» ${ }^{9}$.

En Luis Vives se aprecia una vida que no se deja dominar, una persona que es dueña de su existencia y que lucha constantemente frente a una circunstancia ante la que se niega a doblegarse. A Vives le agradó la holgura, la libertad para hacer su vida conforme a su proyecto y le aterró la sensación de asfixia vital. Es una vida esforzada y noble que rebosa vitalidad pese a lo doloroso de las enfermedades y las amenazas de la época. Cultivó el ingenio como medio del hombre para encontrarse consigo mismo y despegarse del mundo animal, destacó por la profundidad en su pensamiento, por su clarividencia, por su apasionamiento y por mantenerse íntegro en sus principios.

[5] Los escritos de Ortega sobre Vives son «Juan Luis Vives (1492-1540)» (O.C. V, pp. 651-653) y «Juan Luis Vives y su mundo» (O.C. IX, pp. 441-470) «Vives» (O.C. V, pp. 609-622). Las obras de José Ortega y Gasset se citan según la versión en 10 vol. de Taurus/Fundación Ortega y Gasset, Madrid, 2004-2010.

[6] Citado en Peña González, J., Un español en Europa. Una aproximación a Juan Luis Vives. Madrid: Instituto de estudios europeos, 2002, p. 27.

[7] Vives, L., Obras completas, Vol. 1. Madrid: Aguilar, 1947, p. 1200.

[8]Idem.

[9] «La ética y el problema de Dios en Juan Luis Vives», Maestre Sánchez, A., en Anales del Seminario de Historia de la Filosofía, 20 (2003) p. 210. 
Su lema es bien conocido: Sine Querela, sin disputas. Toda una apuesta por el diálogo y la tolerancia con aquellos que piensan diferente. La paz y la concordia serán sus principios y estandartes. Contrario a toda oposición beligerante, buscará siempre una vía intermedia que distienda posiciones enfrentadas.

El Tratado del socorro de los pobres fue escrito por Vives en Brujas y publicado en 1526. Es el primer tratado que lleva la pobreza como centro de toda la obra y responde a una petición de las autoridades de la ciudad flamenca preocupadas por la proliferación del pauperismo en sus tierras. El siglo XVI trajo consigo una gran preocupación por la pobreza, la cual había crecido llamativamente en toda Europa y especialmente en Gran Bretaña. Ante este requerimiento Vives desarrolló un tratado en dos partes bien diferenciadas: una primera parte en la que abordó cuestiones de carácter general sobre la pobreza y sobre la responsabilidad humana con la que sentó las bases de una incipiente antropología de la pobreza y de la marginación; en segundo lugar, Vives fue más allá de las responsabilidades individuales o eclesiales para paliar la pobreza y ofreció un breve tratado de política social en el que reguló las actuaciones que las instituciones cívicas deben poner en funcionamiento para paliar o aliviar la pobreza ${ }^{10}$.

Como buen católico renacentista, la Sagrada Escritura y los clásicos grecolatinos son la principal fuente de inspiración para los argumentos que atraviesan la obra. Por ello cuando inició su trabajo su punto de partida está en el pecado original como fuente de las necesidades humanas y de todos sus males. Por el alejamiento de Dios que explica el Génesis, el ser humano es menesteroso, necesitado y por ello dependiente de los demás, a unos les puede faltar la salud, a otros el dinero, a otros el ingenio, etc. por lo que puede decirse que todos necesitamos de la misericordia de los demás, la cual no se reduce sólo a paliar las necesidades económicas sino cualquier otra necesidad humana. Dios dispuso pues que el hombre necesitara de la ayuda de otro, por ello puede decirse que hacer el bien es natural y la razón para practicarlo no puede reducirse a una contraprestación económica, más bien hay que hacer el bien para alcanzar una vida virtuosa, para acercarse a la verdad y prepararse para la bienaventuranza que es un reino de misericordia. Según Vives, hay ciertos obstáculos que impiden hacer el bien a los que pueden hacerlo son: la envidia, confundir el vicio y la virtud, el dinero, echar la culpa a otros de nuestros defectos

[10] Las propuestas de Vives «se separaron de las formas tradicionales de la práctica de la caridad, orientándolas hacia la municipalización y la burocratización de la asistencia social», en Muñoz Machado, S., Sobre la pobreza y el derecho (Discurso de investidura como Doctor «Honoris Causa» por la Universidad de Valencia), Valencia, 7 de marzo de 2013, p. 25. 
y especialmente la soberbia. Sin embargo, ninguno de ellos puede ser un impedimento para hacer el bien.

Establece Vives en la primera parte un principio fundamental: «que lo que da Dios a cada uno no se lo da para él solo», el cual la doctrina social de la Iglesia ha sabido desarrollar como el destino universal de los bienes. Hay que ser consciente de las necesidades de los hombres y es innegable que hay unos más pobres que otros por lo que se deberá atender con mayor urgencia a quien más lo necesite sin distinción de razas pueblos o naciones, más bien siguiendo la propuesta cristiana de hermandad universal.

En la segunda parte lo fundamental no es tanto lo que debe hacer cada uno sino lo que debe hacer el Estado, por lo que se asientan las bases de una teoría política de la pobreza. Compara Vives el Estado con un cuerpo cuyos diferentes miembros son todos importantes por lo que no debe privarse a ninguno de ellos del sustento vital. La metáfora orgánica sirve pues a Vives para exponer la necesidad de que los pobres sean atendidos también con el mismo derecho que cualquier otro grupo social. En la sociedad la sangre tiene que llegar a todo el cuerpo, no sólo a los miembros más nobles o útiles y para conseguir cuidar de todos hay que recurrir a remedios útiles y permanentes no a ayudas puntuales. Desde el punto de vista cristiano y atendiendo a este razonamiento, la desatención de los pobres es algo vergonzoso.

Pero ¿qué puede hacer el Estado ante el crecimiento de la pobreza? Las propuestas de Vives son sencillas y a la vez muy avanzadas para su época. En primer lugar deben listarse y registrarse todos los menesterosos de las ciudades y clasificarlos según sus necesidades. No se debe permitir a los pobres estar ociosos pues el trabajo es saludable tanto para el cuerpo como para el alma. El trabajo a realizar debe ir acorde con la salud y la edad pero no es admisible el engaño y la picaresca, por lo que serán los médicos los que hagan las valoraciones en cada caso. A los mendigos que son de otra tierra cree Vives que es bueno darles los medios necesarios para poder volver a su casa, en el caso de estar en otra tierra por motivos bélicos conviene ofrecerles refugio.

Tampoco sería bueno tratar a todos por igual pues es necesario distinguir aquellos que siempre han sido pobres de aquellos que estando en una situación acomodada han dilapidado su fortuna con sus vicios: éstos merecen un menor sustento por parte del Estado y un trabajo más duro. Por último, la educación se convierte en la mejor arma según Vives para prevenir la pobreza en general y en mayor medida la pobreza infantil.

¿De dónde pueden obtenerse medios para poner en marcha estas medidas? En primer lugar advierte Vives que es fundamental la administración adecuada y trasparente de los bienes públicos. Estos 
pueden tener como origen tanto una parte de la renta de los más nobles como donaciones; ahora bien, el valenciano hace unas observaciones: Aquellos lugares en los que se atiende a pobres, como por ejemplo hospitales, deben administrarse de forma que intenten ser lo más independientes posible gracias al trabajo de sus miembros. Por otra parte el Estado podría eliminar gastos superfluos como convites, regalos, fiestas anuales, etc.

Es posible que muchos se opongan a estas medidas, afirma Vives, sin embargo no puede ser un obstáculo para desarrollarlas, incluso si los mismos pobres se resisten a aceptarlas, el Estado debe comportarse con ellos como un padre bueno que quiere lo mejor para sus hijos, por lo que no debe dudar en aplicarlas. Todo lo que puede venir de la aplicación de estas medidas es bueno, tanto desde el punto de vista humano como divino. La ciudad ganará en honores, se evitarán los hurtos, mejorará la concordia entre los ciudadanos, existirá mayor seguridad, las conciencias ganarán en quietud y además todos se harán más merecedores de la gloria eterna.

La originalidad de la obra de Vives es relativa, por una parte no puede decirse evidentemente que es el primero que aborda el tema de la pobreza como responsabilidad social, pero es cierto que su trabajo ha quedado en la historia como un hito fundamental y una referencia inexcusable sobre este asunto. La repercusión de este tratado en su época fue notable aunque es necesario distinguir entre su repercusión intelectual y su repercusión política. En lo político unas sesenta ciudades tomaron medidas inspiradas en la obra de Vives ${ }^{11}$ aunque su influencia es quizá más intensa a largo que a corto plazo. La influencia intelectual de la obra fue notable atendiendo a las polémicas generadas ${ }^{12}$, las publicaciones posteriores y la difusión de la misma, tema expuesto magistralmente por la obra de Moreno Gallego La recepción hispana de Juan Luis Vives. Sin embargo no puede negarse el eclipse que en cierto modo ha sufrido históricamente por parte de la obra de Santo Tomás Moro Utopía. Ahora bien, entre ellas hay que destacar la diferencia que se establece en cuanto a la propiedad privada, visiones diferentes que nos dan dos comprensiones de la sociedad

[11] Ver la obra de Moreno Gallego, V., La recepción hispana de Juan Luis Vives. Valencia: Generalitat Valenciana, 2006, p. 442.

[12] Especial malestar crea la obra en el contexto de las órdenes religiosas. El obispo franciscano Nicolás de Bureau tacha la obra de herética y denunciable ante la inquisición y también el agustino Lorenzo de Villavicencio considera el tratado de Vives como pernicioso. La consideración del Erasmismo como una doctrina Luterana por parte de destacados religiosos contaminó la consideración que muchos frailes tenían de Vives por su cercanía al maestro de Rotterdam. Puede verse sobre ello Sebastián Solanes, R.F. y Olucha Feliu, N., «Juan Luis Vives versus Martín Lutero. La respuesta del Humanismo vivesiano a la Reforma luterana», Vivesiana, Universidad de Valencia, Vol. II (2017) pp. 79-90 y también «Apunte sobre escolástica medieval y humanismo: el proceso de Valladolid en una epístola de Juan Luis Vives a Erasmo de Rotterdam (1527)» en AHDE, tomo LXXXV (2015) p. 449-474. 
muy distintas. Si Moro apuesta por la supresión de la propiedad privada en su Utopía, Vives hace una propuesta más realista y menos utópica que ha resultado aceptada en los modelos de Estado actuales por conjugar con cierto éxito la libertad personal y la solidaridad social. Ahora bien ello no es óbice para llamar ladrones a aquellos no son capaces de poner los bienes privados a disposición de los que no tienen nada. Dice Vives: «ladrón es, vuelvo a decir, y robador, todo aquel que desperdicia el dinero en el juego, que lo retiene en su casa amontonado en las arcas, que lo derrama en fiestas y banquetes, el que lo gasta en vestidos muy preciosos o en aparadores llenos de varias piezas de oro y plata, aquel a quien se le pudren en casa los vestidos, los que consumen el caudal en comprar con frecuencia cosas superfluas o inútiles; finalmente, no nos engañemos, todo aquel que no reparte a los pobres lo que sobra de los usos necesarios de la naturaleza» ${ }^{13}$.

El trabajo de Vives es un tratado sobre el pauperismo que da por superada la visión de la pobreza como maldición divina que todavía puede encontrarse en la Edad Media y apuesta por la responsabilidad social como único tratamiento realmente efectivo de la misma. La pobreza es para Vives, siguiendo a Erasmo, un mal social que debe atajarse y la integración social de los pobres es para Vives una prioridad ${ }^{14}$. El ejercicio de la segunda parte se convierte en un proyecto realmente innovador que, a la luz de los Estados actuales del Bienestar, muestra todo su potencial y la enorme originalidad de la propuesta del valenciano. Sin embargo no podemos olvidar que es la propuesta de un cristiano en una sociedad cristiana y su circunstancia es el Renacimiento, lo que visto desde el siglo XXI puede entenderse como una limitación. Como bien ha visto Sebastián Solanes: «Vives plantea el cuidado y socorro de los necesitados como un derecho de los pobres a salir de su pobreza como una exigencia de caridad propia de cristianos y de autoridades de una ciudad cristiana que vive conforme a los preceptos del evangelio cristiano. No lo plantea como un deber de la comunidad política a satisfacer ese derecho de todo ser humano a tener lo mínimo para vivir dignamente, ni mucho menos lo plantea desde la perspectiva de una ética cívica que diga que además de ser un deber, solucionar la pobreza es una exigencia de justicia que afecta a gobernantes y el resto de miembros de una comunidad $\rangle^{15}$. Ahora bien, sin perjuicio de lo dicho no puede negarse que en esta obra, de neta inspiración cristiana, como

[13] Vives, J.L. Tratado del socorro de los pobres. Valencia: Prometeo, 1920, p. 71.

[14] Sobre este aspecto pueden verse las reflexiones de Panea Márquez, J.M., «Juan Luis Vives y la responsabilidad para con los pobres» en Pensamiento, Vol. 73 (2017), n²78, pp. 1173-1196.

[15] Sebastián Solanes, R. F., «La aportación de Juan Luis Vives al estudio de la pobreza. Del socorro de los pobres a la aporofobia», en Vivesiana, Universidad de Valencia, Vol. III (2018) p. 85. 
dice Adela Cortina: «estaba germinando esa Primera Ilustración sobre la Pobreza, e incluso lo que siglos más tarde sería el Estado del Bienestar» ${ }^{16}$.

\section{2.- Francisco de Vitoria y los derechos humanos}

Francisco de Vitoria fue un dominico español, posiblemente nacido en Burgos en 1942, que realizó sus estudios en Paris entre 1509 y 1523, ${ }^{17}$ dónde coincidió con Luis Vives entre 1509 y 1514. En 1516 inicia su docencia en el colegio de Santiago de la Universidad de París y en 1522 obtiene la licenciatura y el doctorado en Teología. Ya de vuelta en España, en 1523 se incorpora como profesor de teología al colegio San Gregorio de Valladolid hasta que en 1526 muere Fr. Pedro de León, catedrático de Prima de teología en la Universidad de Salamanca, y la orden de los dominicos le designa para opositar a la misma. Obtiene la cátedra y el 21 de septiembre se incorpora al claustro universitario en el que permanecerá el resto de su vida académica. Discípulo de Crockaert, introdujo en Salamanca la Suma de Santo Tomás, como este lo había hecho en Paris. Aunque su obra se conoce principalmente a través de los escritos de sus discípulos, ${ }^{18}$ relecciones, la grandeza de su doctrina está hoy en día fuera de toda duda. Maestro de maestros, Vitoria es, junto con Domingo de Soto, uno de los fundadores de la que será conocida como Escuela de Salamanca.

Vitoria fue, pues, teólogo, pero su obra va a penetrar en el Derecho, la Antropología, la Economía o la Política ${ }^{19}$. La razón de todo ello la expone sintéticamente Torrent cuándo afirma que «No debe olvidarse que la obra de Vitoria es esencialmente teológica, en un momento en el que la teología era un conocimiento omnicomprensivo y trascendente». ${ }^{20}$ El propio Vitoria afirma en el comienzo de la relección sobre la potestad civil que «El oficio del teólogo es tan vasto, que ningún argumento, ninguna disputa, ninguna

[16] Cortina, A., Aporofobia, el rechazo al pobre. Barcelona: Paidós, 2017, p.136.

[17] Datos biográficos extraídos de Urdanoz, T., Obras de Francisco de Vitoria. Relecciones teológicas. Madrid: BAC, 1960, 1-84.

[18] Puede consultarse su obra además de la ya citada obra de Urdanoz en la reciente edición crítica publicada por Osuna Fernández, A. (dir.) Francisco de Vitoria, Relección jurídicas y teológicas, 2 vols. Salamanca: San Esteban, 2017.

[19] Baste citar alguno de los artículos que sobre Francisco de Vitoria se han publicado en los últimos años: Cendejas Bueno, J. L. «Justicia, mercado y precio en Francisco de Vitoria», en Revista Empresa y Humanismo XXI, n 1 (2018), pp. 9-38.

[20] Torrent Ruiz, A. J. «Segunda Escolástica Española y renovación en la Ciencia del Derecho en el siglo XVI: un capítulo de los fundamentos del Derecho europeo. I. Francisco de Vitoria, Domingo de Soto», en Teoría e storia del diritto privato, no 6 (2013) pp. 25. 
materia, parecen ajenos a su profesión». ${ }^{21}$ Es claro que ello explica también que en su método se usen a la vez fuentes de revelación y de razón. Se ha destacado que el pensamiento de Vitoria se seculariza al tomar como sujeto de su visión jurídica la comunidad humana y no la cristiandad. Es una visión basada en la igual naturaleza de todo hombre o, como diríamos hoy, en la dignidad de todo ser humano. Esta visión del ser humano como sujeto racional, libre y social, está al base de la reflexión sobre la influencia de Vitoria en el origen de la Ciencia del Derecho Internacional y de la formulación y defensa de los derechos humanos como derechos subjetivos. ${ }^{22}$ Siendo ello cierto, también lo es que la reflexión de Vitoria no deja nunca de ser teológica. La visión que tiene sobre el hombre parte siempre de la visión que tiene sobre Dios. Sus evidentes avances humanistas nunca abandonan el punto central de que todo cuánto hace parte de la reflexión de un Dios creador, causa del hombre. Por tanto el avance en la defensa de los derechos del hombre o en la promoción de una comunidad humana universal, avances humanistas, parten de una filosofía católica teocéntrica, ${ }^{23}$ de una antropología que afirma que la razón humana es capaz de desentrañar lo justo y la voluntad humana, capaz de quererlo. En este mismo sentido, Zorroza pone de relieve que la recuperación del legado de Vitoria ha dejado en evidencia el prejuicio de cierto racionalismo que «estimaba nula la aportación a la ciencia desde posiciones confesionales, pues solo podía hacerse ciencia desde un comienzo netamente racional sin supuestos ni dependencias». ${ }^{24}$ No es por tanto de extrañar que en su libro sobre la influencia de la Iglesia Católica en la construcción de la civilización occidental, Woods dedique un capítulo a los orígenes del Derecho internacional en el que expone sintéticamente como el Padre Francisco de Vitoria sentó las bases de la teoría del Derecho Internacional. ${ }^{25}$

La repercusión de su obra fue inmediata. En primer lugar, en su propia Universidad donde, como se ha dicho, fue uno de los fundadores de la Escuela de Salamanca. Pero, además, en el resto de Universidades, como puso de manifiesto el insigne historiador Hinojosa:

[21] Urdanoz, op. cit., 150.

[22] Ver Cavallar, G. The Rights of Strangers: Theories of International Hospitality, the Global Community and Political Justice since Vitoria. Ashgate: London, 2002, p. 52 (ebook). Tierney, B. The Idea of Natural Rights: Studies on Natural Rights, Natural Law, and Church Law 1150-1625, William B Eerdmans Publishing Company, Grand Rapids, Mi. 1997.

[23] Ver Brufau Prats, J. La Escuela de Salamanca ante el descubrimientod el nuevo mundo. Salamanca: San Esteban, 1989, p. 53 y Torrent, op. cit., 48.

[24] Zorroza, M ${ }^{\text {a }}$ I. «Francisco de Vitoria: actualidad y perspectivas para su estudio», en Azafea. Revista de Filosofía 18 (2016) p. 61.

[25] Woods Jr., Th. E. Cómo la Iglesia construyó la civilización occidental. Madrid: Ciudadela libros, 2007, pp. 171-190. 
Maravilla la rapidez con que se incorporaron a la corriente general de la ciencia europea las doctrinas de Vitoria y la intensidad de la influencia que ejercieron no solo en los países católicos, sino en los protestantes. ${ }^{26}$

Esta repercusión sigue creciendo hoy en día desde planteamientos que en muchos casos son actuales. ${ }^{27}$ Como afirmó en su día Brown, su Derecho de gentes habría de convertirse, con el transcurso del tiempo, en el Derecho Internacional no sólo de la cristiandad, sino del mundo. ${ }^{28}$

Un buen ejemplo de su forma de proceder se refiere a la justificación de una intervención en una comunidad que hoy calificaríamos de soberana. La causa humanitaria es una de las razones por las que Vitoria justifica la intervención militar sobre un pueblo soberano. Para ejemplificar analiza en términos hipotéticos el posible canibalismo de los indios americanos. Si fuera así, ¿estaría justificada la intervención? Cavallar analiza magistralmente esta cuestión poniéndola incluso en relación con los debates actuales sobre el papel de Naciones Unidas en la defensa de los derechos humanos cuando están siendo violados por los propios poderes públicos. En opinión de Cavallar,

I suggest that we acknowledge Vitoria's originality in this respect. He took a first and authentic look at the problem, though those after him made important contributions in thinking the problem over. Vitoria might not have liked to be labelled as an original thinker, and some claim from a contemporary perspective that his original just titles were exactly the ones open to abuse. ${ }^{87}$ In this section, my emphasis is on humanitarian intervention as a problematic right in ius gentium. ${ }^{29}$ (...) Vitoria mentions two conditions that might justify intervention: personal tyranny and tyrannical and oppressive laws against the innocent, such as human sacrifice practised on innocent men or the killing of condemned criminals for cannibalism. ${ }^{30}$

[26] Hinojosa y Naveros, E. de, «Los precursores españoles de Grocio» en AHDE 6 (1929) p. 229.

[27] Así, Cavallar, op. cit., 99 afirma referido al tercer argumento contra el canibalismo, que «This is Vitoria’s strongest and most modern argument».

[28] Brown Scott, J. The Spanish origin of international law: Francisco de Vitoria and his law of nations. Oxford: Clarendon Press, 1934, preface IX: «That there was a Spanish school of international law in the sixteenth century, within forty years after the discovery of America; that the founder of this school was Francisco de Vitoria, prima professor of theology in the University of Salamanca; and that his two 'Relections, De Indis Noviter Inventis' and 'The Iure Belli', set forth his law of nations which was to become the international law not merely of Christendom but of the world at large».

[29] Cavallar, op. cit., 98.

[30] Ibidem, 104. Ver Urdanoz, op. cit., 1050, cuando Vitoria enuncia la quina conclusión: «Los príncipes cristianos pueden hacer la guerra a los bárbaros porque se alimentan de carne humana y sacrifican hombres». 
Veamos pues esta segunda afirmación. La primera parte de la relección sobre la templanza está dedicada a si el hombre está obligado a conservar su vida por el alimento ${ }^{31}$. Es en esta parte cuando se plantea si es lícito comer carne humana. Expone Cavallar que Vitoria da cuatro argumentos contra dicha práctica ${ }^{32}$. Un primer argumento, de orden general, podemos encontrarlo cuándo Victoria se pregunta si hay algún alimento prohibido a los hombres. Cita a Santo Tomás cuándo afirma que «todos los animales están naturalmente sometidos al hombre» ${ }^{33}$. Otros dos podemos encontrarlos en la cuestión tercera ${ }^{34}$ cuándo se hace un enfoque teológico (Eating human flesh is forbidden in divine law ${ }^{35}$ ) o se utilizan argumentos de la razón (Comer carne humana es abominable en naciones civilizadas y humanas; luego es injusto ${ }^{36}$. Encontramos el último argumento cuando Vitoria habla de sacrificios humanos ${ }^{37}$, también puede aplicarse al caso que nos ocupa la afirmación de que «...la vida de un hombre no está en poder de otro hombre, como lo está la de los animales. No es el hombre dueño de su vida ni de la ajena.... ${ }^{38}$.

Creemos que estamos ante un buen ejemplo de la forma de actuar de Vitoria. Una profunda reflexión, que parte de su fe cristiana y en la filosofía religiosa que le inspira, pero que se cuestiona todo y razona sólidos argumentos en la sincera búsqueda de la verdad y la justicia.

\section{El padre Mariana y la cuestión del tiranicidio}

Juan de Mariana es uno de los jesuitas más importantes del siglo de oro español. Es especialmente conocido por ser el autor de una voluminosa historia de España y por ser un autor que defiende abiertamente

[31] Urdanoz, op. cit., 1004.

[32] Cavallar, op. cit., 103.

[33] Urdanoz, op. cit., 1011. Según Cavallar, op. cit, 103: «Humans have a precisely defined position in the order of nature. Their food should be 'confined to organisms which exist on levels of being lower than that of the consumer'».

[34] Urdanoz, op. cit., 1024- 1039.

[35] Ibídem, 1025.

[36] Urdanoz, op. cit., 1026. En palabras de Cavallar, op. cit., 103: «The third argument is that cannibalism is immoral and antisocial. It usually involves homicide and murder, which violate one of the first principles, and undermine the very fabric of any community». Volviendo a Vitoria, un poco más adelante afirma "... una cosa es de derecho natural cuando lo dicta la naturaleza y es común a todos; luego, si todos los hombres tienen esa costumbre por mala y perversa, es que debe serlo por derecho natural», Urdanoz, op. cit., 1027.

[37] Urdanoz, op. cit., 1031-1039.

[38] Ibídem, 1035. Así, explica Cavallar este argumento: Cannibalism constitutes an injury (iniuria) to other men, who cannot renounce their rights and must therefore be helped. 
el tiranicidio. Nació en Talavera de la Reina en 1536, profesando en la compañía de Jesús en 1554 y fue profesor de las universidades de Roma, Palermo y París. Sorprendentemente y por razones de salud se retiró a Toledo a componer las obras que le darían fama. Ayudó en algunos dictámenes de la inquisición y él mismo fue procesado sin consecuencias ${ }^{39}$ pero no por sus opiniones sobre le tiranicidio, sino por denunciar públicamente que la alteración de la moneda por parte del gobierno era una clase de robo. Murió en $1624^{40}$.

Mariana es mundialmente conocido por estar asociado su nombres a la cuestión del tiranicidio ${ }^{41}$, que no expone en un tratado de moral sino en un tratado de educación de príncipes pedido por encargo ${ }^{42}$. Este detalle, que se suele obviar, es especialmente relevante para entender la expresión retórica de alguna de sus ideas. Hace un tratamiento magistral de la cuestión: primero distingue a los reyes de los tiranos (cap. V); narra la muerte de Enrique III a manos de Jacobo Clement para a continuación exponer los argumentos en contra y a favor del tiranicidio, sentando la propia doctrina (cap. VI) y termina examinando la cuestión de si es lícito envenenar al tirano. Sorprende un poco que tras haber establecido que es lícito matar al tirano en ciertas condiciones niegue la posibilidad de envenenarlo con dolo porque en tal caso el tirano estaría suicidándose.

Tras exponer sus ideas políticas, más cercanas al republicanismo clásico que al liberalismo moderno ${ }^{43}$, Mariana diferencia con precisión al rey del tirano. El monarca ideal trata a los súbditos como hijos y no como esclavos, no sube los impuestos sin necesidad ni se cree dueño del Estado, vive modestamente, predica con el ejemplo y no se rodea de aduladores. El tirano en cambio no llega legítimamente al poder sino mediante intrigas empleando el poder en beneficio propio. Le temen los buenos en lugar de los malos, e impone tributos nuevos cada día. En síntesis: el buen rey teme por sus súbditos mientras que el tirano teme por sí mismo.

Vista la diferencia, se impone la pregunta de si es lícito o no matar al tirano. Y Mariana comienza narrando magistralmente el asesinato de

[39] Fernández de la Mora, G., «El proceso del padre Mariana», en Revista de estudios Políticos (Nueva Época), 79, Enero-Marzo (1993).

[40] Para profundizar véase la síntesis, Crespo, M., El P. Juan de Mariana. Aproximación a su vida y obra. Fundación Ignacio de Larramendi, 2009.

[41] Y sin embargo no todos lo reconocen debidamente. Véase la escasa atención que le dedica por ejemplo Franklin L. Ford, Political Murder: From Tyrannicide to Terrorism, Harvard, 1985, p. 157.

[42] Juan de Mariana, Del rey y la institución real, en la Biblioteca de autores españoles (BAE), vol. 31. Madrid: Real Academia Española, 1950.

[43] Rubio-Carracedo, J. «Ciudadanos y príncipes. El concepto de ciudadanía activa en Juan de Marina», en Revista de estudios políticos (Nueva época), 138 (2007) pp. 129-156. 
Enrique III de Francia, odiado por el pueblo por cambiar de religión y por asesinar a los nobles contrarios, a manos de Jacobo Clement, un estudiante de teología que había oído en clase que era lícito matar al tirano.

Hay quien niega el derecho a matar al tirano y Mariana expone sus argumentos. Hay ejemplos bíblicos que lo desaconsejan (David no mató ni permitió que mataran a Saul, que se había convertido en tirano); el ejemplo de los primeros cristianos que sufrieron persecución a manos de un poder tiránico también lo desaconseja. San Pablo afirma que hay que someterse a la autoridad (sin especificar si es autoridad legítima o no). Además, quizá matando al tirano empeore la situación. Habría más bien que preguntarse si la crueldad del rey no viene por la insubordinación de los súbditos. En último lugar: si fuera legítimo matar al rey por la misma razón cualquiera podría matar a quien considerase malo y se desataría el caos. A todas estas objeciones Mariana les da cumplida respuesta más adelante. Sin embargo también hay opiniones a favor del tiranicidio. El argumento principal es porque «la dignidad real tiene su origen en la voluntad de la república». Además, los tiranicidas son considerados héroes por parte del pueblo, como muestran algunos ejemplos clásicos. Por último, si nos defendemos de una mala bestia cuanto más nos defenderemos de los tiranos, aun a costa de la propia vida.

Vistos los argumentos contrapuestos Mariana expone la cuestión desde su punto de vista sin salirse del planteamiento canónico que recibe de la tradición, como veremos. Hay dos tipos de tirano: el que se apodera a la fuerza del puesto y el que aunque sea un rey legítimo se comporta ilegítimamente. Si se trata de un rey que accede ilegítimamente al poder todos están de acuerdo en que puede ser despojado por cualquiera de la corona e incluso de la vida, tal como muestran los ejemplos bíblicos de Aod y Eglón (Jue. 3, 12 y ss). Pero si se trata de un rey legítimo que ha accedido al poder legítimamente (por herencia o por voluntad del pueblo) entonces el pueblo le ha de soportar mientras no quebrante las leyes. Si se trata de un rey que quebranta las leyes (roba o hace violencia al pueblo, no respeta lo santo, etc.), hay que amonestarle, y si no se enmienda pasar a la guerra con él. Para ello habrá que reunirse previamente el pueblo o los notables, y si la tiranía ha llegado al punto de que no hay libertad de reunión cualquiera podría atentar contra la vida del rey. Importa notar que la calificación de tirano no se circunscribe a una apreciación personal sino que ha de ser algo percibido así por todos. La cuestión así planteada es mucho más matizada de lo que el tópico transmitido sobre Mariana pretende:

- Tirano que accede ilegítimamente: Cualquiera puede matarlo.

- Tirano que accede legítimamente pero se comporta mal.

- Si no quebranta las leyes: Hay que aguantarlo. 
- Si quebranta las leyes:

- Si tiene autoridad superior, avísese a dicha autoridad.

- Si no tiene autoridad superior:

- Si deja que el pueblo se reúna: Reunión y amonestación.

- Si se enmienda: aguantar al rey.

- Si no se enmienda: decláresele pena de muerte.

- Si no deja que el pueblo se reúna: mátelo cualquiera.

¿De dónde bebe Mariana su doctrina? La cuestión se remonta a Platón, Aristóteles o Cicerón ${ }^{44}$. En los concilios visigodos se había condenado duramente el tiranicidio fundándose en que hay un pacto que liga al rey con los súbditos ${ }^{45}$ (el detalle del pacto es importante, porque algunos autores lo recogerán posteriormente); pero sin embargo en la doctrina de San Isidoro de Sevilla encontramos el fundamento filosófico de su legitimidad: rex eris si juste feceris, et si non, non eris $^{46}$. En Juan de Salísbury ${ }^{47}$ aparece tratada por primera vez la cuestión de modo extenso y favorable al tirancidio, pero será Santo Tomás (De regimine principorum) quien asiente doctrina sobre el particular, defendiendo la legitimidad del tiranicidio de origen, y la ilegitimidad del tiranicidio de ejercicio.

En la misma época de Mariana otros autores trataban la cuestión ${ }^{48}$. Fray Alonso de Castro (Adversus omnes haereses, París, 1584) cataloga cualquier tipo de tiranicidio como herejía condenable. Fray Domingo de Soto, dominico de la escuela de Salamanca como Vitoria, (Iustitia et iure, Salamanca, 1556) añade al tratamiento de Santo Tomás que si uno es tirano porque se comporta como tal y atenta contra la vida de un ciudadano este puede matar al tirano defendiéndose. El agustino Fray Juan Márquez

[44] H. G. Ganss, «The Jesuits and Tyrannicide. Did the jesuits teach the doctrine of assassinating tyrans», en American Catholic Quarterly Review, 27 (1902) pp. 334-351.

[45] Concilio IV de Toledo, num. 75.

[46] Recogida en cierto sentido por el mismo IV concilio de Toledo: «Y acerca de los futuros reyes, promulgamos esta determinación: que si alguna de ellos en contra de la reverencia debida a las leyes, ejerciere sobre el pueblo un poder despótico con autoridad soberbia y regia altanería, entre delitos, crímenes y ambiciones, sea condenado con sentencia de antema, por Cristo Señor, y sea separado y juzgado por Dios porque se atrevió a obrar malvadamente y llevar el reino a la ruina». Véase más en profundidad Orlandis, J. «En torno a la noción visigoda de tiranía», en Anuario de historia del derecho español (1959) pp. 5-43.

[47] Merle, A., «El De rege de Juan de Mariana (1599) y la cuestión del tiranicidio: ¿un discurso de ruptura?», en Criticón 120-121 (2015) pp. 89-102.

[48] Autores expuestos con precisión en Solana, M., «La resistencia a la tiranía según la doctrina de los tratadistas del siglo de oro español», en Acción Española, Tomos 6 y 7, núm. 34 a 37, (1933). Mucha mayor precisión podemos encontrarla en la tesis de Centenera, F., El tiranicidio en los escritos de Juan de Mariana: un estudio sobre uno de los referentes más extremos de la cuestión, Universidad de Alcalá de Henares, 2005. Disponible en https://ebuah.uah.es/dspace/handle/10017/445 
(Gobernador cristiano deducido... Salamanca, 1612), que podía conocer ya las tesis de Mariana, dice también que si se trata de un tirano que se queda con los bienes de los súbditos no se le puede matar, pero si se trata de un tirano asesino que mata a su pueblo sí que se le podría matar.

Especialmente relevantes para la cuestión por ser compañeros de orden (jesuitas) son Luis de Molina y Francisco Suárez. Molina (De Iustitia et iure, 1593-1600) distingue los dos tipos de tirano y afirma que además de matar al tirano por el origen al tirano de ejercicio se le puede matar como mucho si me intenta matar a mí, como legítima defensa. Suárez, el doctor eximio, trata el tema en su Defensio Fidei Catholica (cap. IV, lib. VI. Coimbra, 1613) y ya conocía las ideas de Mariana ${ }^{49}$. Afirma que el rey no puede ser muerto por autoridad privada de nadie: no se le puede matar como castigo (porque el súbdito no tiene legitimidad para castigar al rey); tampoco se le puede matar para defender bienes de fortuna inferiores a la vida; se le podría matar a lo sumo para defender la propia vida o la vida de la república si no hay otra alternativa, si no haya poderes superiores a los que apelar, si la tiranía es evidente, si no se siguen males mayores, si no hay un juramento explícito sobre esto (a menos que sea un juramento injusto), etc.

Las repercusiones de las doctrinas de Mariana pueden rastrearse en hechos y en teorías políticas. En la cuestión de los hechos es bien sabido que el gobierno francés apuntó directamente a Mariana como instigador de la muerte de Enrique IV a manos de un asesino, razón por la cual los escritos del jesuita fueron quemados en París aunque la relación entre el asesino y los escritos de Mariana no está probada ${ }^{50}$. Los escritos de Mariana junto con los de Suárez también fueron incinerados simbólicamente en Inglaterra por miedo a los hechos que pudieran seguirse a partir de ahí. Aunque Mariana sea el más radical de cuantos autores trataran la cuestión queremos recordar que no la expone en un tratado de moral sino en un tratado sobre la educación del príncipe. Por ello no es justa la acusación de Bayle $^{51}$ y otros, de convertir a Mariana en un autor que invita a la sedición $\mathrm{y}$ al asesinato del monarca, sin matizar las posturas.

Más interesante es la relación de las ideas de Mariana con determinadas ideologías políticas posteriores. A Mariana se le ha situado como un precedente de la derecha liberal, entendiendo por liberalismo la oposición a cualquier forma de tiranía en favor de la libertad de los individuos; mejor

[49] Una comparación en el artículo de Font, P., «Suárez, Mariana y el tiranicidio: Convergencias, divergencias y silencios estratégicos», en Cuadernos salmantinos de filosofía, 44 (2017) pp. 11-34.

[50] Centenera, Op. Cit., p. 422 y siguientes.

[51] Carracedo, Op. Cit., p. 155. 
aún se le ha señalado como precedente del republicanismo por su noción de ciudadanía activa, precisamente a propósito del tiranicidio; e incluso como precursor de cierta izquierda revolucionaria ${ }^{52}$. La desobediencia civil o la objeción de conciencia serían desarrollos muy suaves de la doctrina del tiranicidio. Más interesante que esto nos parece que el carácter cristiano de nuestro autor le permite establecer una distinción entre el origen del poder y el depositario del poder político: esto permite que cuando el poder se corrompe haya instancias distintas del poder que permitan cuestionarlo o incluso quitarlo de en medio. De lo contrario, si no se distingue el depositario de poder del origen del poder, sin ninguna instancia externa al poder que sirva como norma (Dios, la Ley natural, etc.) entonces tenemos serios problemas para cuestionar un poder absoluto que se ha corrompido: sea el poder de uno, sea el poder de todo el pueblo ${ }^{53}$.

\section{Conclusión}

Hemos presentado estos autores con la doble intención de mostrar, en primer lugar, que las ideas religiosas no siempre están determinadas por las condiciones materiales de la existencia sino que a veces son ellas mismas las que determinan estas mismas condiciones; y en segundo lugar que la religión no es sólo una fuerza conservadora sino que también es una fuerza progresiva que propicia cambios benéficos para la sociedad. Juan Luis Vives propuso una atención integral y planificada al agudo problema de la pobreza sistémica de las sociedades de su tiempo: los pobres nos conciernen porque son hermanos nuestros según el dogma cristiano. Francisco de Vitoria propuso que las naciones se concertasen no en base a la ley del más fuerte sino en base al ideal de justicia cuya superioridad y bondad arraiga en la bondad e inteligencia de un benevolente Dios creador. Juan de Mariana defendió con argumentos bíblicos y teológicos (además de filosóficos) la conveniencia de eliminar al tirano que desangra a su pueblo porque su autoridad no es absoluta sino que está subordinada a Dios y su ley, debiéndose al pueblo a cuyo servicio está.

[52] Herrero, M., «El padre Mariana y el tiranicidio», en Torre de los Lujanes, 65 (2009) especialmente pp. 104-107.

[53] Un desarrollo interesante de esta doctrina en Negro Pavón, D., «Derecho de resistencia y tiranía», en Logos. Anales del seminario de metafísica, num. Extra (1992) pp. 683-708. 


\section{Bibliografía}

Abellán J. L., Historia crítica del pensamiento español Vol. II. Madrid: Espasa-Calpe, 1979.

Brown Scott, J. The Spanish origin of international law: Francisco de Vitoria and his law of nations. Oxford: Clarendon Press, 1934.

Brufau Prats, J. La Escuela de Salamanca ante el descubrimientod el nuevo mundo. Salamanca: San Esteban, 1989.

Cavallar, G. The Rights of Strangers: Theories of International Hospitality, the Global Community and Political Justice since Vitoria. London: Ashgate, 2002.

Cendejas Bueno, J. L. "Justicia, mercado y precio en Francisco de Vitoria», en Revista Empresa y Humanismo XXI, n 1 (2018), pp. 9-38.

Centenera, F., El tiranicidio en los escritos de Juan de Mariana: un estudio sobre uno de los referentes más extremos de la cuestión, Universidad de Alcalá de Henares, 2005. Disponible en https://ebuah.uah.es/dspace/ handle/10017/445

Cortina, A., Aporofobia, el rechazo al pobre. Barcelona: Paidós, 2017.

Crespo, M., El P. Juan de Mariana. Aproximación a su vida y obra. Fundación Ignacio de Larramendi, 2009.

Dawkins, R., El espejismo de Dios. Madrid: Espasa, 2007.

Fernández de la Mora, G., «El proceso del padre Mariana», en Revista de estudios Políticos (Nueva Época), 79, Enero-Marzo (1993).

Font, P., «Suárez, Mariana y el tiranicidio: Convergencias, divergencias y silencios estratégicos», en Cuadernos salmantinos de filosofía, 44 (2017) pp. 11-34.

Franklin L. Ford, Political Murder: From Tyrannicide to Terrorism. Harvard, 1985.

Ganss, H. G. «The Jesuits and Tyrannicide. Did the jesuits teach the doctrine of assassinating tyrans», en American Catholic Quarterly Review, 27 (1902) pp. 334-351.

Guy, A. Los filósofos españoles de ayer y de hoy. Buenos Aires: Losada, 1966.

Harris, S., El fin de la fe. Religión, terror y futuro de la razón. Madrid: Paradigma, 2007.

Herrero, M., «El padre Mariana y el tiranicidio», en Torre de los Lujanes, 65 (2009) pp. 103-121. 
Hinojosa y Naveros, E. de, «Los precursores españoles de Grocio» en $A H D E$ 6 (1929).

Hitchens, C., Dios no es bueno. Barcelona: Debate, 2009.

Marx, C., Prólogo a la Contribución a la crítica de la economía política, en Marxists Internet Archive, marzo de 2001.

Ortega y Gasset J, Obras completas, 10 vol. Madrid: Taurus/Fundación Ortega y Gasset, 2004-2010.

Peña González, J., Un español en Europa. Una aproximación a Juan Luis Vives. Madrid: Instituto de estudios europeos, 2002.

Maestre Sánchez, A., "La ética y el problema de Dios en Juan Luis Vives», en Anales del Seminario de Historia de la Filosofía, 20 (2003) pp. 181-245. Mariana, Juan de., Del rey y la institución real, en Biblioteca de autores españoles (BAE), vol. 31. Madrid: Real Academia Española, 1950.

Merle, A., "El De rege de Juan de Mariana (1599) y la cuestión del tiranicidio: ¿un discurso de ruptura?», en Criticón 120-121 (2015) pp. 89102.

Monterde, J. C., "Apunte sobre escolástica medieval y humanismo: el proceso de Valladolid en una epístola de Juan Luis Vives a Erasmo de Rotterdam (1527)» en AHDE, tomo LXXXV (2015) p. 449-474.

Moreno Gallego, V., La recepción hispana de Juan Luis Vives. Valencia: Generalitat Valenciana, 2006.

Muñoz Machado, S., Sobre la pobreza y el derecho (Discurso de investidura como Doctor "Honoris Causa» por la Universidad de Valencia), Valencia, 7 de marzo de 2013.

Negro Pavón, D., «Derecho de resistencia y tiranía», en Logos. Anales del seminario de metafísica, num. Extra (1992) pp. 683-708.

Orlandis, J. «En torno a la noción visigoda de tiranía», en Anuario de historia del derecho español (1959) pp. 5-43.

Osuna Fernández, A. (dir.) Francisco de Vitoria, Relección jurídicas y teológicas, 2 vols. Salamanca: San Esteban, 2017.

Panea Márquez, J.M., «Juan Luis Vives y la responsabilidad para con los pobres» en Pensamiento, Vol. 73, n 278 (2017), pp. 1173-1196.

Rubio-Carracedo, J. «Ciudadanos y príncipes. El concepto de ciudadanía activa en Juan de Marina", en Revista de estudios políticos (Nueva época), 138 (2007) pp. 129-156.

Sebastián Solanes, R.F. y Olucha Feliu, N., «Juan Luis Vives versus Martín Lutero. La respuesta del Humanismo vivesiano a la Reforma luterana», Vivesiana, Universidad de Valencia, Vol. II (2017) pp. 79-90. 
Sebastián Solanes, R. F., «La aportación de Juan Luis Vives al estudio de la pobreza. Del socorro de los pobres a la aporofobia», en Vivesiana, Universidad de Valencia, Vol. III (2018).

Solana, M., "La resistencia a la tiranía según la doctrina de los tratadistas del siglo de oro español», en Acción Española, Tomos 6 y 7, núm. 34 a 37, (1933).

Tierney, B. The Idea of Natural Rights: Studies on Natural Rights, Natural Law, and Church Law 1150-1625, William B. Eerdmans Publishing Company, Grand Rapids, Mi. 1997.

Torrent Ruiz, A. J. «Segunda Escolástica Española y renovación en la Ciencia del Derecho en el siglo XVI: un capítulo de los fundamentos del Derecho europeo. I. Francisco de Vitoria, Domingo de Soto», en Teoría e storia del diritto privato, $\mathrm{n}^{\circ} 6$ (2013).

Urdanoz, T., Obras de Francisco de Vitoria. Relecciones teológicas. Madrid: BAC, 1960.

Vives, L., Obras completas, Vol. 1. Madrid: Aguilar, 1947.

Vives, J.L. Tratado del socorro de los pobres. Valencia: Prometeo, 1920.

Weber, M., La ética protestante y el espíritu del capitalismo. Madrid: Akal, 1998.

Woods Jr., Th. E. Cómo la Iglesia construyó la civilización occidental. Madrid: Ciudadela libros, 2007.

Zorroza, $M^{\mathrm{a}}$ I. «Francisco de Vitoria: actualidad y perspectivas para su estudio», en Azafea. Revista de Filosofía 18 (2016). 